\title{
Subband Adaptive Filtering for Acoustic Echo Control Using Allpass Polyphase IIR Filterbanks
}

\author{
Patrick A. Naylor, Member, IEEE, Oğuz Tanrıkulu, Member, IEEE, \\ and Anthony G. Constantinides, Fellow, IEEE
}

\begin{abstract}
Adaptive filtering in subbands is an attractive alternative to full-band schemes in many applications because of the potential for faster convergence and lower computational cost. However, the analysis of a signal into a subband representation and the synthesis back into its original full-band form carries three main penalties. These are that 1) the subsampling process often introduces aliasing, 2) the subband analysis and synthesis processes carry a computational overhead, thereby reducing the gain in efficiency, and 3) the subband analysis and synthesis processes introduce delay into the signal path. In this paper, a subband scheme is presented that aims to minimize these penalties, thereby allowing the potential advantages of the subband approach to be more fully realized. The scheme is based on infinite impulse response (IIR) filterbanks, formed from allpass polyphase filters, which exhibit very high quality filtering compared to typical finite impulse response (FIR) implementations, have relatively low complexity, introduce a limited degree of phase distortion and have low delay. The scheme, in conjunction with normalized least mean squares (NLMS) adaptive filters, is tested in an acoustic echo control application and shown to give better convergence, lower delay, and lower computational cost than a comparable FIR subband scheme.
\end{abstract}

Index Terms - Adaptive filters, echo cancellation, filterbanks.

\section{INTRODUCTION}

$\mathbf{I}$ $\mathrm{N}$ THIS paper we describe the application of subband adaptive filtering to the problem of acoustic echo control. The primary contribution and aim of the work is to reduce some of the limitations present in typical subband approaches based on finite impulse response (FIR) filterbanks. To this end, we introduce a subband structure based on allpass polyphase filterbanks and examine its characteristics in the context of acoustic echo control for hands-free terminals. A principal application of this work is to mobile hands-free telephones for use on networks such as GSM.

In subband adaptive filtering, adaptive filters are applied in each of $N(N \geq 2)$ frequency subbands [1], [2]. Here we consider adaptive filters based on the least mean squares (LMS) algorithm [3], [4]. The potential advantages of subband adaptive filtering are a reduction in computational complexity and improved convergence speed. The computational saving comes about because, for critical subsampling in which the

Manuscript received July 26, 1995; revised February 21, 1997. This work was supported in part by CEC ESPRIT Project 6166 (FREETEL). The associate editor coordinating the review of this manuscript and approving it for publication was Dr. Dennis R. Morgan.

The authors are with the Department of Electrical and Electronic Engineering, Imperial College of Science, Technology and Medicine, London SW7 2BT, U.K. (e-mail: p.naylor@ic.ac.uk).

Publisher Item Identifier S 1063-6676(98)01686-1.
Nyquist sampling criterion is just satisfied in each subband, the sampling rate in each of the $N$ subbands is $1 / N$ the original rate while the required length of the adaptive filters in each subband is $1 / N$ the length of the equivalent fullband adaptive filter. Consequently, although the number of taps in the adaptive filtering is not reduced, only $1 / N$ of the tap computations are required for each output sample, leading to a reduction in complexity by a factor of $N$. This reduction is only approached in practice, since the filterbanks present a computational overhead that is desirable to minimize. This is one factor that motivated our introduction of allpass polyphase filterbanks, since they are computationally efficient. The improvement in convergence speed of the LMS algorithm applied in subbands comes about because the eigenvalue spread, $\tilde{\lambda}$, for the subband signals is normally less than the eigenvalue spread for the original signal when the original signal is colored, as in our case of speech. The quantity $\tilde{\lambda}$ is defined as the ratio between the largest and smallest eigenvalue of the signal autocorrelation matrix, $\mathbf{R}$. It can be shown [3] that the convergence speed of LMS degrades as $\tilde{\lambda}$ increases, and, hence, we can expect that the subband LMS adaptive filters will converge in fewer iterations than an equivalent full-band case. The value of $\tilde{\lambda}$ affects the convergence of the system's impulse response more than the minimization of the residual echo signal when the input signal is not white noise.

These two factors-lower complexity and faster convergence-have motivated the use of subband adaptive filtering for acoustic echo control in hands-free terminals such as telephones and conferencing systems. Here it is necessary to identify a nonstationary impulse response, of the order of 10-200 ms in length, in real time. This is the impulse response from the loudspeaker to the microphone of the terminal operating in, say, a room or a car. The identified impulse response can be used to cancel the acoustic echo of far-end speech [5] as shown in Fig. 1.

Subband analysis and synthesis is often performed using multirate filterbanks [6], [7]. When the filterbank bandlimiting is nonideal and the subsampling is critical, the analysis bank causes aliasing of the subband signals. This aliasing can be cancelled in the synthesis bank when certain conditions are met by the synthesis filters and in the subband processing [7]. However, even if the aliasing is cancelled in this way, it is still present in the subband adaptive filter input signals and, consequently, the adaptive filters are perturbed and the performance of the overall system is reduced [2]. The severity of the aliasing problem increases with the number of subbands, 


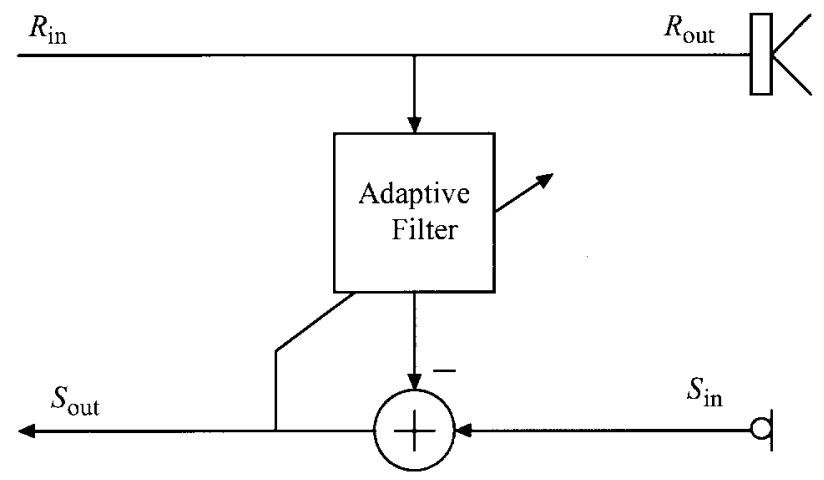

Fig. 1. Schematic diagram of a simple acoustic echo controller.

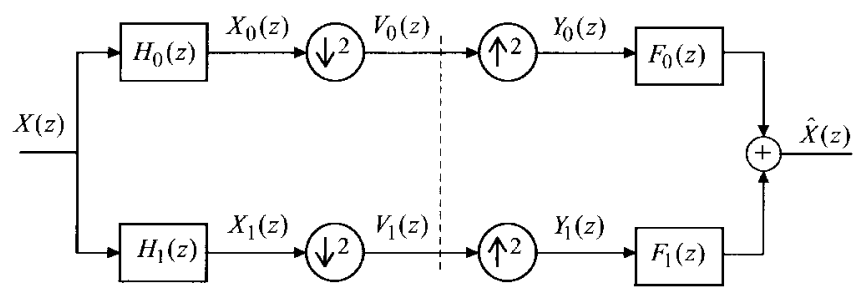

Fig. 2. Two-band cascaded analysis and synthesis filterbanks.

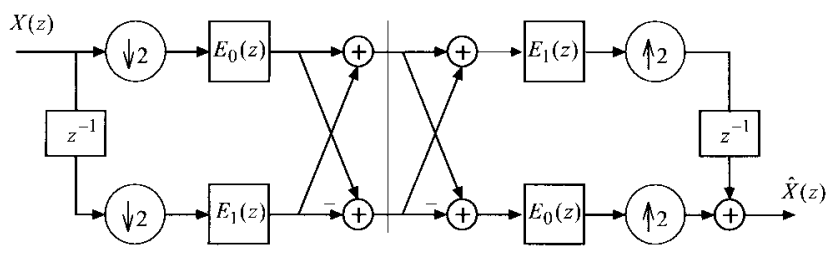

Fig. 3. Two-band cascaded analysis and synthesis polyphase filterbanks.

especially when a binary tree structure of half-band systems is used, and therefore, the improvements in performance that would otherwise follow from increasing the number of subbands is limited. Practical implementations of subband adaptive filtering therefore look for a compromise between the negative effect of aliasing and the benefits in performance and complexity. In previous work, for example [8], the filterbanks employed for this application are FIR quadrature mirror filters (QMF's) [6]. Such filterbanks normally require long FIR filters to obtain sufficient interband discrimination and to reduce aliasing to low levels. Such filters introduce substantial delay into the signal path and are relatively costly to implement. In practical terms, the stopband attenuation is limited by the real-time computational and delay constraints on the length of the FIR filters and, as a consequence, the aliasing problem can be severe for implementable systems. This is a second factor that motivated our introduction of allpass polyphase (AP) infinite impulse response (IIR) filterbanks since, even with short filters, they achieve very high discrimination and can therefore be expected to yield lower levels of aliasing of the subband signals.

Several solutions to the aliasing problem have already been proposed in the literature. These include

1) noncritical subsampling [9], [10] in which the sample rate is reduced by less than the critical factor;

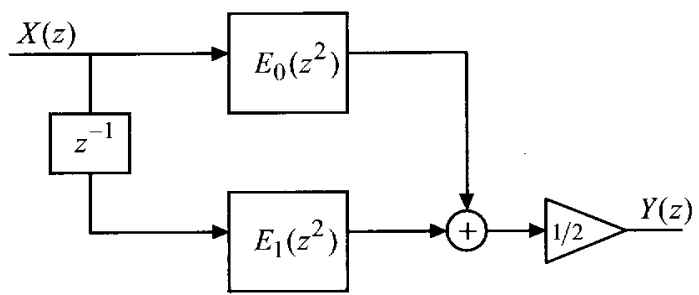

Fig. 4. Allpass polyphase implementation of the IIR filter $H(z)$.

2) the application of cross-adaptive filters [1] between the subbands to explicitly filter the aliasing components;

3) overbandlimiting the signal in each subband so that energy nulls are created in the spectrum at frequencies where aliasing would otherwise be generated [11].

All three proposed methods of solution carry penalties as reported in [2]; 1) has an increased computational cost, 2) has been found to have problematic convergence speed, and 3) causes spectral distortions.

The method we present here is a novel application of allpass polyphase IIR filterbanks that gives very high-quality bandlimiting performance with low computational cost [7]. Being IIR, their delay is relatively short compared to FIR filters of comparable magnitude response, but their phase response is nonlinear. This latter effect could be a disadvantage in certain applications, but has been found not to be a problem in the acoustic echo control application of interest here. Furthermore, it will be seen that the phase is "near-linear" for most of the passband of the filters and that subsequent application of narrowband notch filter preprocessing can be effective in reducing residual aliasing components as well as nonlinear phase effects. The allpass polyphase structures have a particular advantage over other forms of IIR structures in that they are very tolerant of rounding in finite precision implementations, since they are formed from cascades of firstorder sections [12], [13].

The remainder of the paper is structured as follows. Section II briefly reviews some of the relevant theory of multirate filterbanks. In Section III, allpass polyphase IIR filters are reviewed from the point of view of their application to multirate filterbanks. In Section IV, the application of the allpass polyphase filterbanks to subband adaptive filtering is considered and compared to an FIR QMF system and a full-band approach. An overbandlimiting approach using efficient notch filters is presented in Section V. Methods using oversampled FIR filterbanks are viable alternatives for comparison but are beyond the scope of the present paper and are discussed in [14]. Simulation results are given in Section VI and final conclusions are drawn in Section VII.

\section{MaXimally Decimated ANALYSIS AND SyNTHESIS FILTERBANKS}

Here we consider the use of adaptive filters in each of $N$ subbands. When the subband analysis and synthesis filterbanks decimate by $N$, then the decimation is said to be critical or maximal. Fig. 2 shows a two-band analysis bank and synthesis bank connected in cascade. 

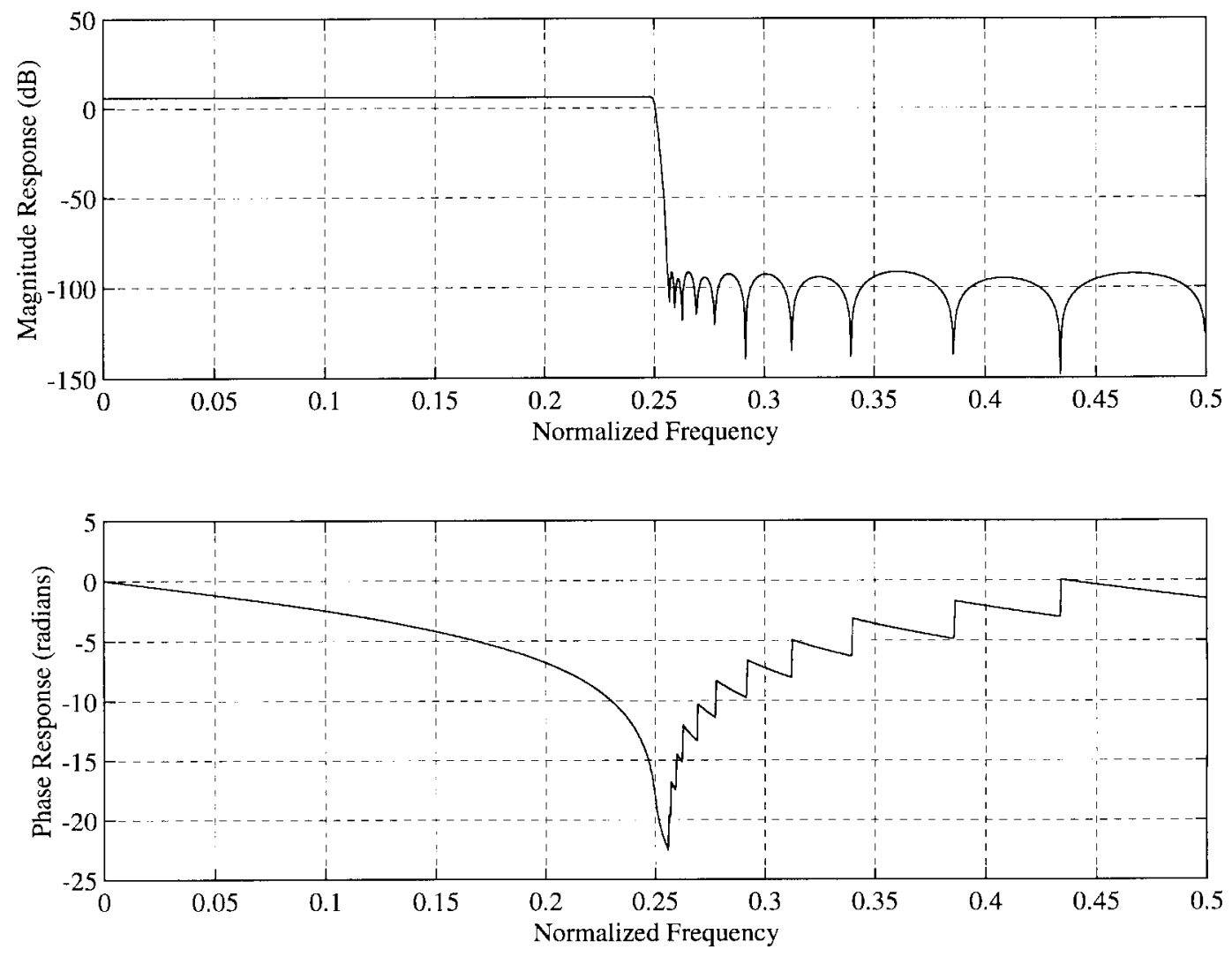

Fig. 5. Frequency response of the allpass polyphase lowpass prototype filter.

For an input signal $x(n)$ and output $\hat{x}(n)$, we can write [7] that their $z$-transforms are related by

$$
\begin{aligned}
\hat{X}(z) & =\frac{1}{2} \mathbf{F}(z) \mathbf{H}(z) \mathbf{X}(z) \\
\mathbf{F}(z) & =\left[\begin{array}{ll}
F_{0}(z) & F_{1}(z)
\end{array}\right] \\
\mathbf{H}(z) & =\left[\begin{array}{cc}
H_{0}(z) & H_{1}(z) \\
H_{0}(-z) & H_{1}(-z)
\end{array}\right] \quad \mathbf{X}(z)=\left[\begin{array}{c}
X(z) \\
X(-z)
\end{array}\right]
\end{aligned}
$$

where $\mathbf{H}(z)$ is known as the aliasing component matrix. When $H_{1}(z)$ is chosen as $H_{1}(z)=H_{0}(-z)$ the magnitude responses of the filters $H_{0}(z)$ and $H_{1}(z)$ are mirror images around $\omega=\pi / 2$ since $\left|H_{1}\left(e^{j \omega}\right)\right|=\left|H_{0}\left(e^{j(\pi-\omega)}\right)\right|$ and the filterbank is QMF.

Terms in $X(-z)$ in the reconstructed signal $\hat{X}(z)$ represent undesired aliasing which can be cancelled after synthesis by careful choice of $\mathbf{F}(z)$, namely

$$
F_{0}(z)=H_{1}(-z) \quad F_{1}(z)=-H_{0}(-z) .
$$

Thus, it is possible to design filterbanks that generate aliased subband signals such that the aliasing can be completely cancelled during synthesis.

Efficient polyphase implementations of filterbanks have been presented, for example, in [7], [15] and [16], in which all filtering is done at the lowest possible sampling rate. The bandlimiting filters $H_{0}(z)$ and $H_{1}(z)$ can be expressed in polyphase form (type 1), for the case $N=2$, as

$$
\begin{aligned}
& H_{0}(z)=E_{0}\left(z^{2}\right)+z^{-1} E_{1}\left(z^{2}\right) \\
& H_{1}(z)=E_{0}\left(z^{2}\right)-z^{-1} E_{1}\left(z^{2}\right) .
\end{aligned}
$$

By application of the noble identities, [7] the order of the two operations in multirate filterbanks (bandlimiting filtering followed by subsampling) can be reversed as shown in Fig. 3 .

\section{AllPass Polyphase IIR STRUCtURES FOR SUBbAND ANALYSIS AND SYNTHESIS}

As seen above, when the analysis filters in critically sampled filterbanks have finite transition bandwidths, the subband signals generated contain aliasing that can be perfectly cancelled in the synthesis bank. However, when adaptive filters are inserted into each subband, the aliasing cancellation properties of the filterbank may be perturbed and the performance of the adaptive algorithms may be degraded by the presence of the aliasing signal components [1], [2], [7]. For a system $S(z)$ to be identified and a filter prototype $H(z)$, an optimum model for $S(z)$ when $N=2$ is given [2] to within a delay and a multiplicative constant by

$$
\left[\begin{array}{l}
H^{2}(z) S(z)-H^{2}(-z) S(-z) H(z) H(-z)[S(-z)-S(z)] \\
H(z) H(-z)[S(z)-S(-z)] H^{2}(z) S(-z)-H^{2}(-z) S(z)
\end{array}\right] .
$$

The diagonal terms of this square model matrix represent the direct (adaptive) filtering whereas the off-diagonal elements represent cross-filtering between bands. Systems using such cross-adaptive filters have been studied [1], [2] and found to exhibit relatively slow convergence behavior.

In this work, we explore an alternative approach to the square model matrix (4) as follows. If the off-diagonal terms are to be ignored, as is often the case, then a sufficient 

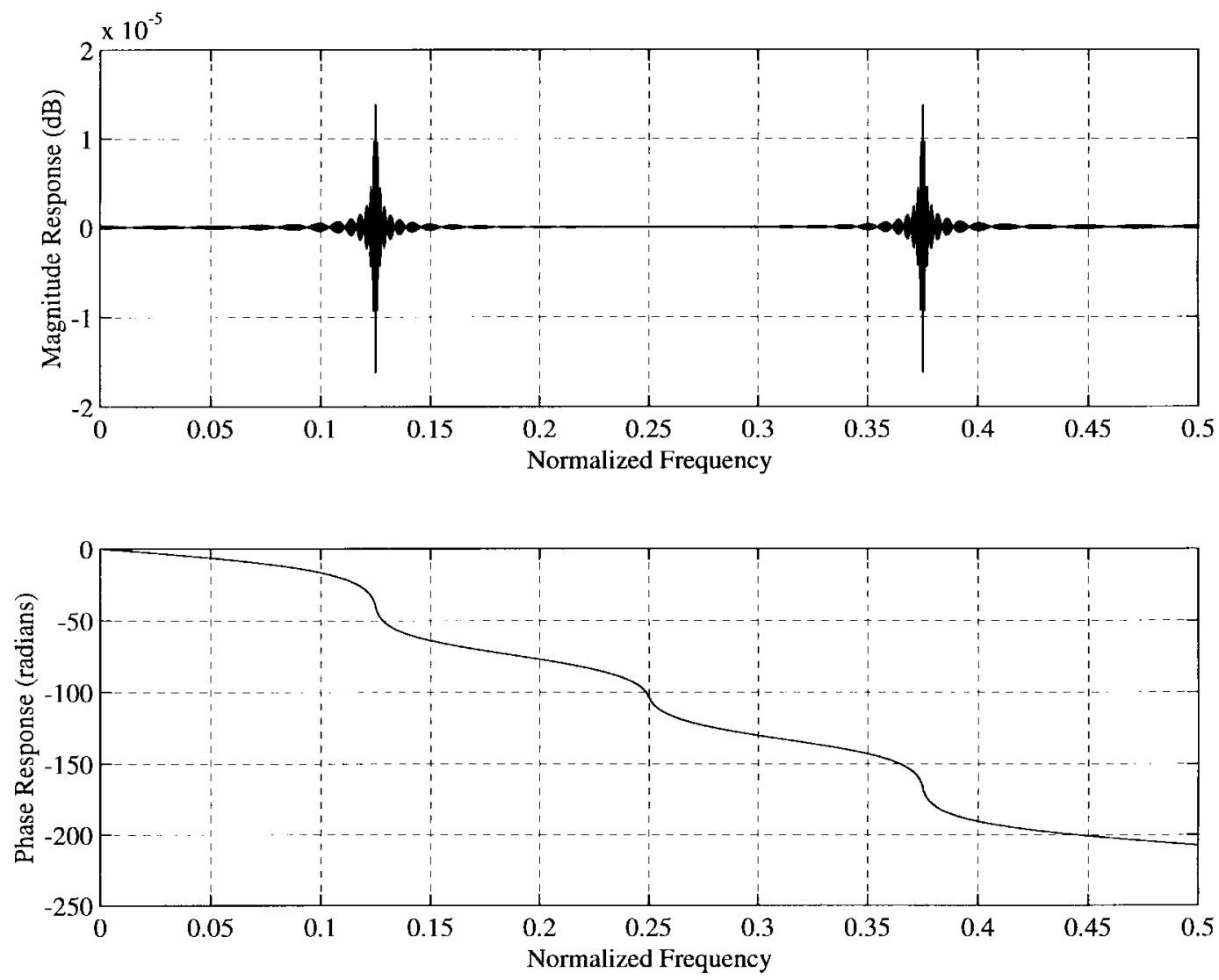

Fig. 6. Reconstruction quality of the polyphase two-band filterbank.

condition to obtain a zero error in each subband of a two-band system is that

$$
H(z) H(-z)=0
$$

Therefore, an alternative to cross-adaptive filtering is to use a filter $H(z)$ such that (5) is satisfied to a close approximation. A possible choice for filter $H(z)$ is a high-quality IIR filter implemented in allpass polyphase form as described, for example, by Valenzuela [12] and Vaidyanathan [7]. Such filters have several desirable properties such as outstanding selectivity, low computational cost, and low delay. These filters can be used to produce multirate filterbanks with zero amplitude distortion in the synthesized signal [7]. The form of the polyphase implementation of the filter $H(z)$ is shown diagramatically in Fig. 4 and is given by

$$
\begin{aligned}
H(z) & =1 / 2\left(E_{0}\left(z^{2}\right)+z^{-1} E_{1}\left(z^{2}\right)\right) \\
E_{k}(z) & =\prod_{i=0}^{N_{k}-1} \frac{\alpha_{k, i}+z^{-1}}{1+\alpha_{k, i} z^{-1}} \quad k=0,1
\end{aligned}
$$

where $\alpha_{k, i}$ is the $i$ th real, constant coefficient of allpass filters $E_{k}(z) . N_{k}$ is the number of coefficients in the $k$ th phase. We limit the discussion to first-order allpass sections, since higher order allpass functions can be built from products of such firstorder filters. Furthermore, to maintain the performance of the filters in fixed point implementation it is advantageous to use cascaded first-order sections [17].
In [2], Gilloire and Vetterli show that, when the filterbank is lossless, mean square error minimization of the individual residuals following adaptive filtering in each subband is equivalent to minimization of the overall residual after the synthesis bank. This is verified in [2] for the FIR QMF case for which the filterbank can be seen as a process that preserves $L_{2}$ norms. It is straightforward to extend this result to the IIR case using $H(z)$ of the form (6) since

$$
\begin{aligned}
& H(z) H\left(z^{-1}\right)+H(-z) H\left(-z^{-1}\right) \\
& =\frac{1}{2}\left(\frac{\alpha_{0}+z^{-2}}{1+\alpha_{0} z^{-2}} \cdot \frac{\alpha_{0}+z^{2}}{1+\alpha_{0} z^{2}}+\frac{\alpha_{1}+z^{-2}}{1+\alpha_{1} z^{-2}} \cdot \frac{\alpha_{1}+z^{2}}{1+\alpha_{1} z^{2}}\right)=1
\end{aligned}
$$

so that allpass polyphase filterbanks are lossless, and we can justify the use of the subband residuals in the minimization procedures.

Design procedures have been presented by, for example, Valenzuela [12], Harris et al. [18], and Vaidyanathan [7] for allpass polyphase filters implemented as cascades of firstorder filters. In Fig. 5, the frequency response achieved using [18] is given for the two-phase case $N_{0}=6, N_{1}=5$. The frequency axis is shown normalized with respect to the sampling frequency. The intrinsic gain of the filter is two, though this is usually normalized, the passband is flat, the stopband attenuation is close to $100 \mathrm{~dB}$ and, on closer inspection, the normalized transition bandwidth (specified from $-3 \mathrm{~dB}$ of passband gain to $-100 \mathrm{~dB}$ ) is 0.0125 . However, such filters 

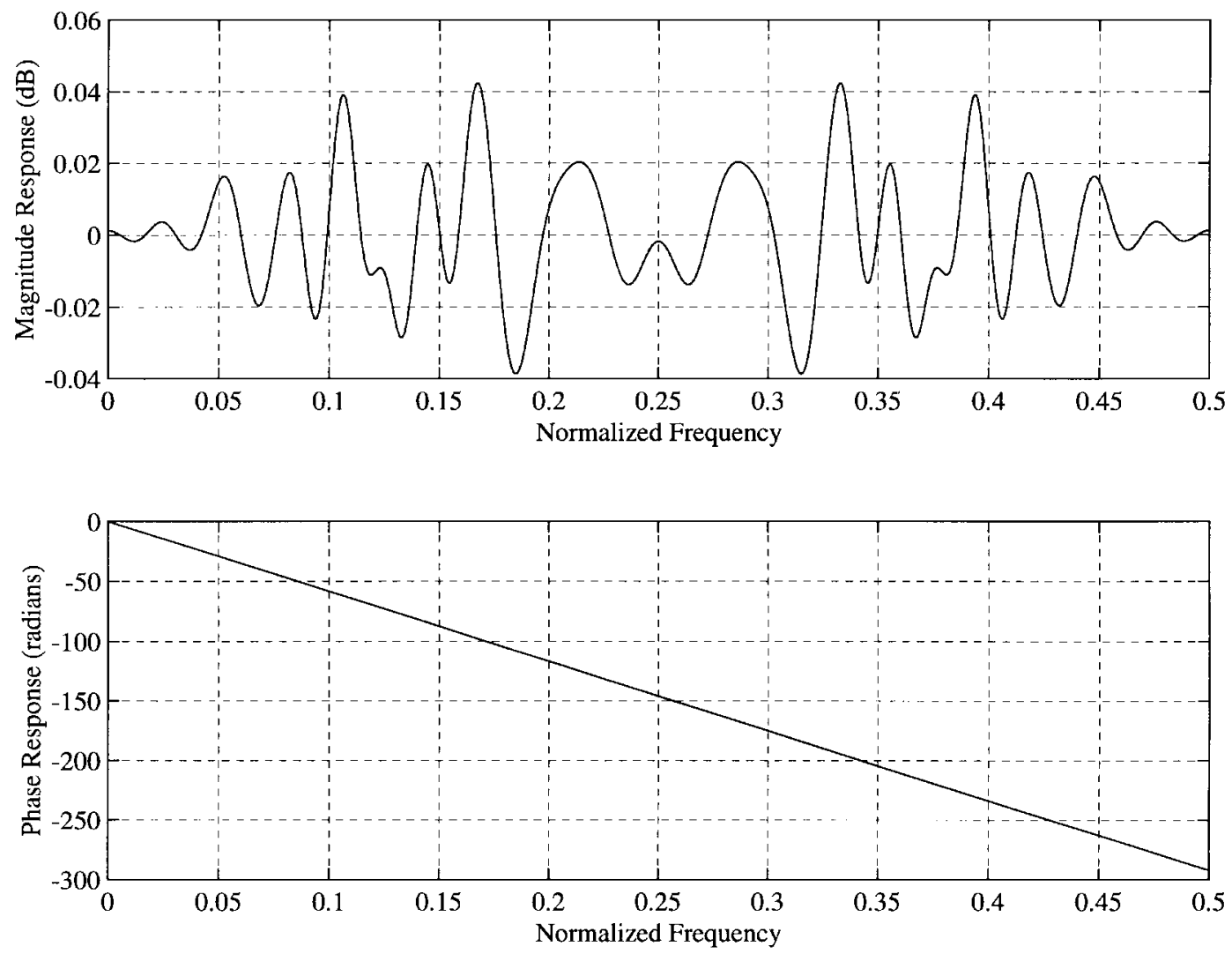

Fig. 7. Frequency response of cascaded analysis-synthesis four-band FIR filterbank.

have a gain of $-3 \mathrm{~dB}$ at a frequency of $\pi / 2$ relative to the passband. This implies that a significant fraction of the (small) transition band will cause aliasing after critical subsampling.

In the two-band polyphase filterbank of Fig. 3, the filtering is based on the prototype filter $H(z)$ of the form given in (6), with frequency response shown in Fig. 5, such that equivalently, with reference to Fig. 2,

$$
H_{0}(z)=H(z), \quad H_{1}(z)=H(-z) .
$$

Fig. 6 shows the frequency response $\hat{X}(z) / X(z)$ of the cascade connected analysis and synthesis banks for a four-band structure formed using a binary tree of two-band structures. From this result, we can observe effectively perfect reconstruction in the magnitude response and the nonlinear phase response as predicted. The phase response can be seen to be "near-linear" except in the region of the band interface.

For comparison, an FIR filterbank using 32-tap FIR filters after Johnston (type 32-D) [16] has also been tested. The frequency response of the cascade connected analysis and synthesis banks for the four-band FIR structure, formed using a binary tree of two-band structures, is shown in Fig. 7. The choice of the number of taps for the FIR case is limited in a practical system by the allowable delay for the network of interest. In the case of GSM networks, 32-taps seems to be a reasonable compromise for a four-band system with $8 \mathrm{kHz}$ sampling frequency. In such a system using a binary tree of two-band structures, the delay is due to a 32-tap filter operating at the original sampling rate followed by a 32-tap filter operating at half the original rate for both the analysis and the synthesis filterbanks. This gives close to $12 \mathrm{~ms}$ of the allowable $90 \mathrm{~ms}$ delay for GSM, with the remaining time being available for coding and transmission delays. This constraint on the amount of delay that can be introduced by the filterbank is an important motivating factor for the introduction of IIR filterbanks in this work, and becomes even more important if the FIR filter lengths are increased so as to obtain better discrimination. A comparison of the group delay of the FIR QMF and allpass polyphase IIR four-band filterbanks, measured at the output of the cascade connected analysis and synthesis banks, is given in Fig. 8 assuming a sampling frequency of $8 \mathrm{kHz}$. This shows the constant $12 \mathrm{~ms}$ delay of the FIR case and the delay of the IIR case to be between $2.5 \mathrm{~ms}$ and $5 \mathrm{~ms}$ except near the band edges, where the phase response nonlinearities are large. The effect of the large group delays at the band edges can be subsequently reduced by the notch filter preprocessing described later in Section V.

The complexity for the analysis filterbanks is given in Table I in terms of the number of multiplies for two-, four-, and eight-band systems. A typical echo canceller requires two such banks plus one synthesis bank with identical complexity. For the FIR filterbanks, an efficient implementation [7] is assumed, exploiting both the presence of decimators and the symmetry relationship of the highpass and lowpass filters.

\section{Subband Adaptive Filtering}

We now consider the application of adaptive filters in each of the subbands resulting from allpass polyphase IIR filter- 


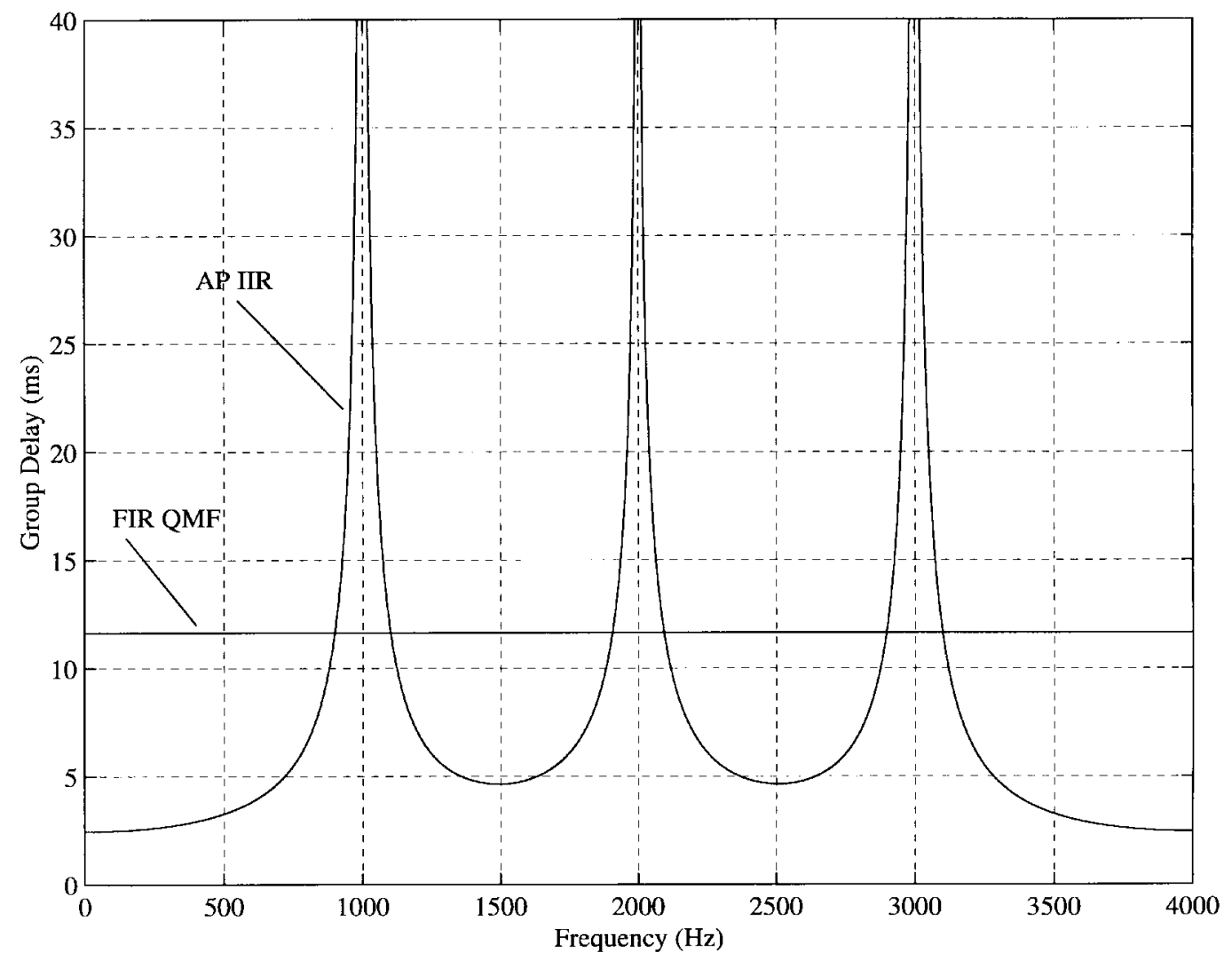

Fig. 8. Comparison of group delay for four-band allpass polyphase IIR (AP IIR) and FIR QMF filterbanks using 8 kHz sampling frequency.

banks. We focus on two questions. First we ask, "What effect does the nonlinear phase distortion have on the performance of the adaptive filters," and, second, "What are the aliasing effects in this case compared to an FIR QMF filterbank?"

To investigate the nonlinear-phase issue, we begin by considering the LMS algorithm, since it is often employed in this context either directly or, as in this work, in the normalized form. For a transversal adaptive filter with weights $w_{0}(n), w_{1}(n), \cdots, w_{M-1}(n)$, zero-mean wide-sense stationary tap inputs $u(n), u(n-1), u(n-2), \cdots, u(n-M+1)$ and desired signal $d(n)$, the mean squared error at time $n$ between the adaptive filter output and the desired signal is given by [3]

$$
J(n)=\sigma_{d}^{2}-\mathbf{w}^{H}(n) \mathbf{p}-\mathbf{p}^{H} \mathbf{w}(n)+\mathbf{w}^{H}(n) \mathbf{R} \mathbf{w}(n)
$$

where $\sigma_{d}^{2}$ is the variance of the desired response, $\mathbf{p}$ is the cross-correlation between the tap-input vector $\mathbf{u}(n)$ and the desired response, $\mathbf{R}$ is the correlation matrix of the tap-input vector, and $(\cdot)^{H}$ indicates Hermitian transpose. Such secondorder signal statistics are phase blind [19] so that if phase distortion is introduced prior to the application of adaptive filtering, the mean squared error resulting at any time $n$ is not perturbed by the phase distortion. This point can be developed by considering the cross-correlation vector $\mathbf{p}$ since this features in a wide variety of adaptive processes. To do this, we consider the system of Fig. 9 in which $Q(z)$ represents an unknown system to be identified by adaptive system identification. The filterbank operations are modeled by $P(z)$. Both $Q(z)$ and $P(z)$ are assumed linear time invariant. The cross power spectral density between the signals $y(n)$ and $z(n)$ can be
TABLE I

COMPLEXITy OF THE FIR AND IIR ANALYSIS (OR SyNTHESIS) FiLterbanK

\begin{tabular}{l|c|c|c|c}
\cline { 3 - 5 } \multicolumn{2}{c|}{} & 2-band & 4-band & 8-band \\
\hline $\begin{array}{l}\text { Number of multiplies per } \\
\text { sample }\end{array}$ & FIR & 16 & 32 & 48 \\
\cline { 2 - 5 } & IIR & 5.5 & 8.25 & 9.625 \\
\hline
\end{tabular}

shown [20] to be given by

$$
S_{y z}\left(e^{j \theta}\right)=\left|P\left(e^{j \theta}\right)\right|^{2} \cdot Q\left(e^{-j \theta}\right) \cdot S_{x}\left(e^{j \theta}\right)
$$

from which it can be seen that the cross-power spectral density is independent of the phase of $P(z)$. Hence, if $P(z)$ is a nonlinear-phase subband analysis filter, its phase response will not perturb the cross spectrum between the desired response and the tap input vector within each subband. This point is seen immediately when the linear time-invariant nature of the filters in Fig. 9 is used to allow the order of $Q(z)$ and $P(z)$ in the lower branch to be swapped and, hence, show that nonlinearities in the filterbanks can be viewed as identical preprocessing operations on both the input signal and desired response. It is therefore concluded that the application of nonlinear-phase subband analysis filters does not perturb adaptive filtering in the subbands.

We now investigate the effect of the magnitude of the offdiagonal terms in (4) by studying the two filterbanks described in Section III. We investigate the magnitude of $H(z) H(-z)$ to 


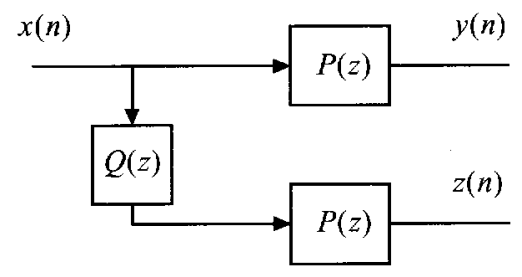

Fig. 9. Model for the investigation of the effect of nonlinear phase distortion.

study the validity of (5), the so-called diagonal approximation [1].

Fig. 10 shows the result of computing the magnitude of $H(z) H(-z)$ for both the filterbank cases. It can be seen that, for the optimized FIR case studied here, the quantity $H(z) H(-z)$ is relatively large (around $40 \mathrm{~dB}$ above the quantization floor of a 16-b system) at all frequencies so that aliasing errors can be expected over the entire band because of the relatively poor stopband attenuation, which is limited by the number of taps of the filter. For the allpass polyphase filter, the quantity $H(z) H(-z)$ is found to be close to zero (the quantization floor of a 16-b system, say) except near the band edges, and so it can be expected that aliasing effects will be small in this case except in a narrow band of frequencies near the band edges where significant aliasing could be expected.

\section{NOTCH FILTERING}

As has been seen in the previous section, the nature of aliasing in allpass polyphase IIR subband signals is narrowband and concentrated around the subband edges. In acoustic echo cancellation the subjective quality of the residual echo signal after processing is very important and audible distortions are undesirable. For the FIR QMF filter, the aliasing components are almost evenly distributed in the spectrum and therefore, it is difficult to discriminate these components. However, for the allpass polyphase IIR filter, the aliasing components affect only a narrow band and can, potentially, be removed by filtering at the cost of introducing a relatively small amount of distortion. An effective way of removing narrowband aliasing effects is to preprocess the loudspeaker and microphone signals ( $R_{\text {out }}$ and $S_{\text {out }}$ in Fig. 1 ) so that the parts of the spectrum that would cause aliasing are sufficiently attenuated over narrow frequency bands. Clearly, the microphone signal must be distorted as little as possible so that the speech signal from the near-end speaker is not audibly distorted after passing through the echo cancellation system. A cost-effective solution is to use notch filters to produce spectral "gaps" at the subband edges. In this work, very sharp notch filters have been implemented using filter transformations of an allpass polyphase IIR lowpass prototype. By starting with a lowpass IIR filter of the form

$$
N\left(z^{-1}\right)=\frac{1}{2}\left(\frac{\beta_{1}+z^{-2}}{1+\beta_{1} z^{-2}}+z^{-1} \frac{\beta_{2}+z^{-2}}{1+\beta_{2} z^{-2}}\right)
$$

with a lower stopband frequency at $\omega_{s}$, the desired notch filter for a system with four subbands can be obtained by applying the following spectral transformations:

$$
\begin{aligned}
& z^{-1} \leftarrow-\frac{z^{-2}-\frac{2 \theta k}{k+1} z^{-1}+\frac{k-1}{k+1}}{\frac{k-1}{k+1} z^{-2}-\frac{2 \theta k}{k+1} z^{-1}+1} \\
& z^{-1} \leftarrow z^{-4}
\end{aligned}
$$

where

$$
\begin{aligned}
& \theta=\frac{\cos \left(\frac{\omega_{2}+\omega_{1}}{2} T\right)}{\cos \left(\frac{\omega_{2}-\omega_{1}}{2} T\right)} \\
& k=\cot \left(\frac{\omega_{2}-\omega_{1}}{2} T\right) \tan \left(\frac{\omega_{s} T}{2}\right) .
\end{aligned}
$$

The first spectral transformation (12) maps $N\left(z^{-1}\right)$ to a bandpass filter with lower and upper stopband frequencies at $\omega_{1}$ and $\omega_{2}$, which are chosen to produce narrow stopbands, or notches, centered at frequencies of zero and $\pi$. The second transformation (13) is for the purpose of repeating the spectral notches at each interface of the four subbands. The amplitude response of such a notch filter is shown in Fig. 11.

This notch filter has interesting properties. The bandwidth of the notches can be controlled with the first spectral transformation. For practical purposes, a narrow bandwidth notch is sufficient to remove the narrowband aliasing in the allpass polyphase IIR filterbank and, thus, there is no audible distortion on the microphone signal. Although a null is obtained at the frequency of the subband edge, the attenuation over the bandwidth of the notch is around $-23 \mathrm{~dB}$, which was found to be adequate in the simulations performed. A disadvantage of this scheme is the delay associated with the high order of the notch filter after the second frequency transformation. The advantages of this scheme are that 1) the number of multiplications required is very low as only a few numerator and denominator coefficients are nonzero after the transformation (13), and 2) it is suitable for fixed-point implementation, since it can be constructed from a cascade of structures that are robust to rounding effects [17].

\section{Simulations}

To investigate the above issues in a practical sense, acoustic echo control experiments have been performed. These experiments compare the performance of the allpass polyphase structure and the FIR QMF filterbank approaches against a baseline method of a full-band NLMS [3] adaptive echo canceller.

For the FIR case, the subband analysis and synthesis are performed using a system built as a binary tree of alias cancelling, linear phase FIR QMF two-band filterbanks in the form of Fig. 2 with

$$
\begin{aligned}
H(z) & =\sum_{i=0}^{N} b_{i} z^{-i} \\
H_{0}(z) & =H(z) \quad H_{1}(z)=H(-z) \\
F_{0}(z) & =H_{0}(z) \quad F_{1}(z)=-H_{1}(z)
\end{aligned}
$$

for which $H(z)$ is the Johnston type 32-D filter as described in Section III. For the IIR case, a binary tree, allpass polyphase IIR filterbank was used in which $H(z)$ is of the form of (6) 


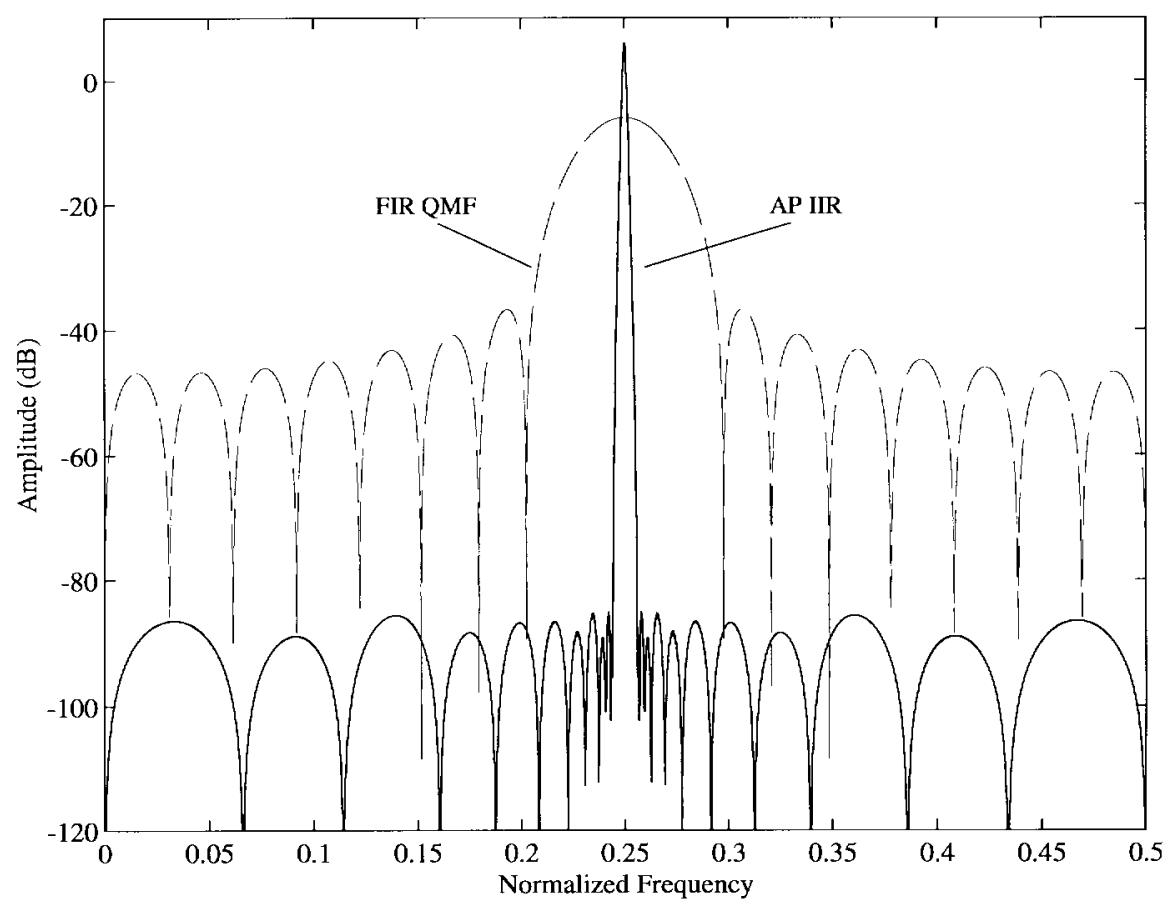

Fig. 10. Cross filter magnitude spectra for FIR QMF filterbank (dashed line) and allpass polyphase IIR filterbank (solid line).

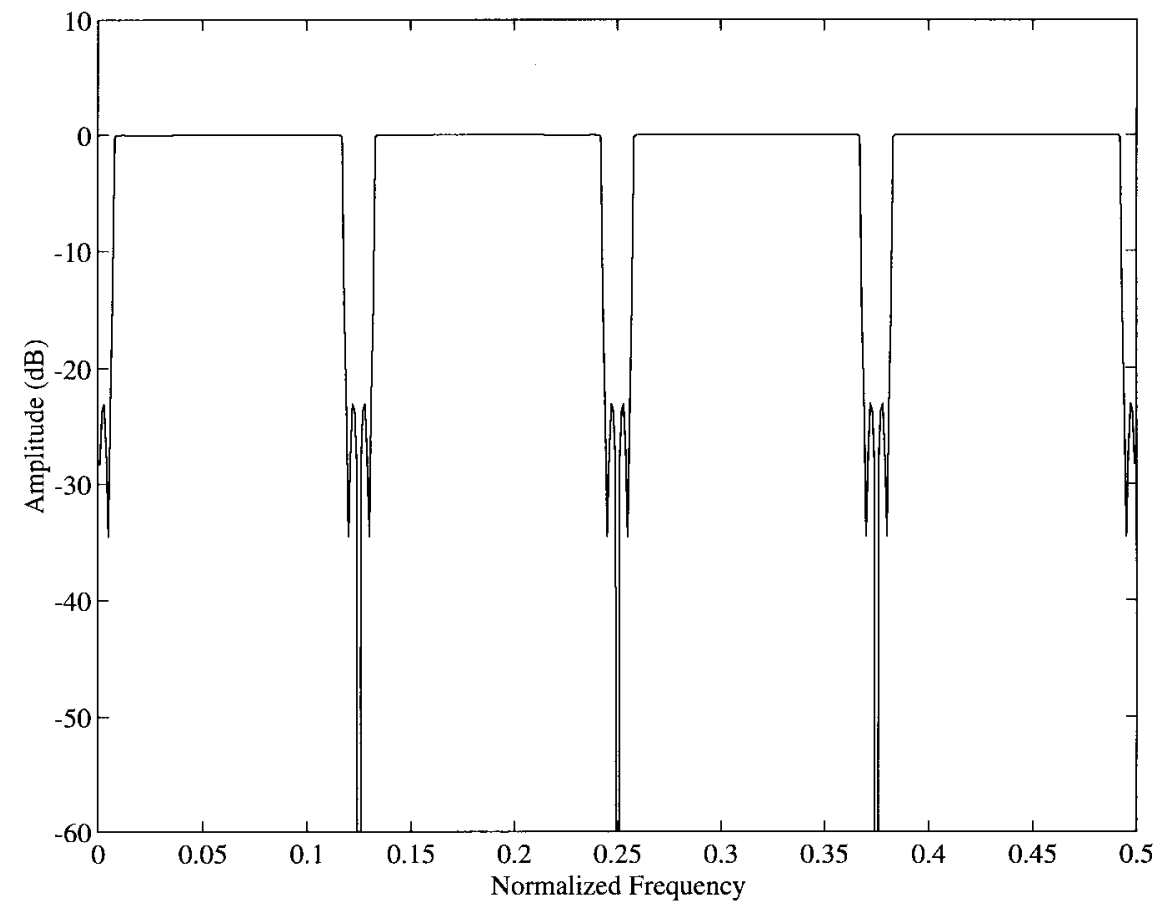

Fig. 11. Amplitude spectrum of the notch filter.

with $N_{0}=6, N_{1}=5$ and for which the frequency response of the equivalent lowpass prototype filter is given in Fig. 5.

In the subband approaches, the NLMS algorithm was applied independently in each subband. The step-size of the NLMS algorithm was determined independently for each subband, proportional to the energy in that subband [1], and was kept the same for both FIR and IIR systems. In both cases, the adaptive filter was of length 512 taps and the taps were divided equally among all subbands, i.e., 128 taps in each of four subbands. For the baseline full-band NLMS approach, the step-size was determined in the same way and, again, 512 taps were used.

The test data consists of 19 independent recordings of the USASI noise signal [21] recorded from a hands-free mobile 


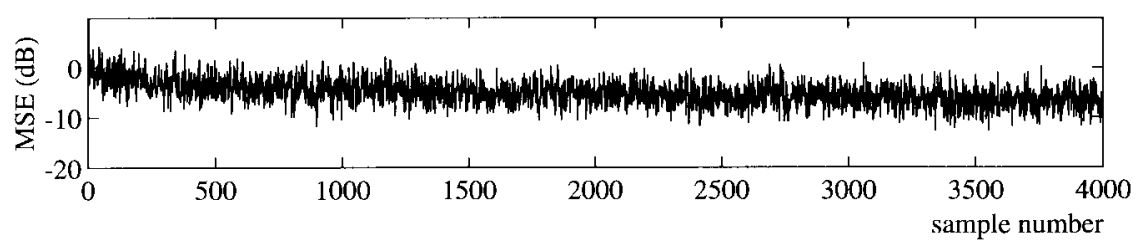

(a)

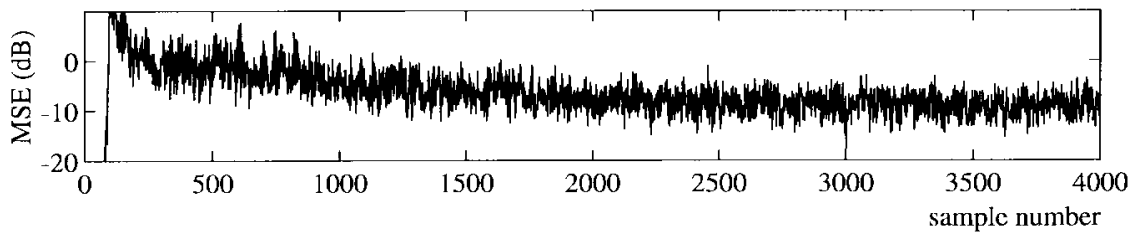

(b)

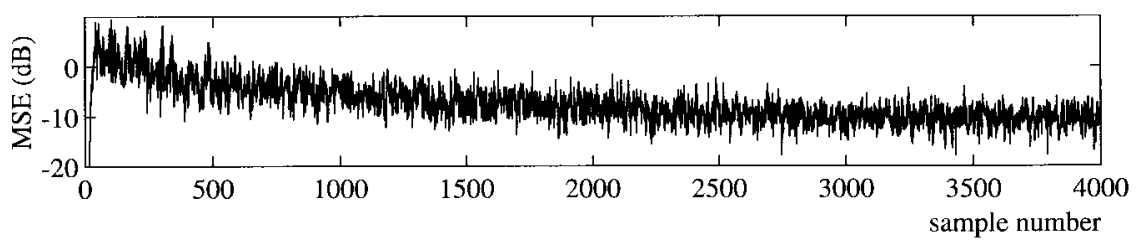

(c)

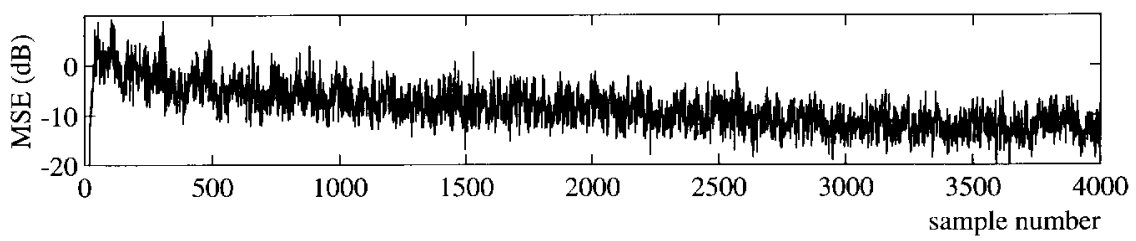

(d)

Fig. 12. MSE performance for USASI input signals. (a) Full-band. (b) four-band FIR filterbank. (c) Four-band allpass polyphase IIR filterbank. (d) Four-band allpass polyphase IIR filterbank with notch.

telephone situated in a small office. The test results presented are averaged over the ensemble. Additional tests on recordings of speech signals from the same setup are also reported below.

Fig. 12(a)-(c) shows the mean square error (MSE) at the output of the echo cancellers in $\mathrm{dB}$ relative to the mean power of the microphone signal for USASI noise input. It can be seen from these results that the FIR filterbank and the IIR filterbank echo cancellers have approximately equal initial convergence and final misadjustment properties, reaching around $10 \mathrm{~dB}$ of cancellation after about 2000 samples. Both the subband approaches are better than the full-band approach as shown in Fig. 12(a). The additional delay in the FIR case can be clearly seen. An alternative presentation of these results can be seen in Fig. 13, which shows the ensemble averaged segmental echo return loss enhancement (ERLE). The segmental ERLE over segment $n$ is defined here, using the notation of Fig. 1, as

$$
\mathrm{ERLE}_{n}=10 \log _{10}\left(\frac{\sum_{i=0}^{M-1} S_{\text {in }}^{2}(n M+i)}{\sum_{i=0}^{M-1} S_{\text {out }}^{2}(n M+i)}\right) \quad M=256 .
$$

Again, it can be seen from the ERLE results that the IIR approach gives approximately the same performance as the FIR approach even though it has lower complexity and delay. This result underlines the practical potential of the allpass polyphase IIR filterbank for real-time implementations of acoustic echo control in which complexity and delay are subject to tight constraints that are not easily met with the full-band or FIR QMF subband 512-tap adaptive structures.

It has been observed [2] that, in the mean, the FIR approach degrades in performance as the number of subbands increases. This is based on the principle that, as the number of subbands increases, so do the errors due to aliasing. We suggest that such errors increase less in the allpass polyphase IIR case because of the highly selective nature of the filters employed and because of the cross-magnitude characteristics shown in Fig. 10. To investigate the aliasing effects, the individual subband error signals were studied for the four-band canceller. Amplitude spectra of the subband error signals are plotted in Fig. 14 for the FIR case and Fig. 15 for the allpass polyphase IIR case. These spectral plots were obtained by operating the cancellers using white noise signals recorded from the same handsfree telephone and then performing a 512-point FFT on each subband's error signal after convergence. Five independent tests were done and the results averaged in the spectral domain. It can be seen that the allpass polyphase IIR case exhibits sharper peaks in the error amplitude spectrum close to $2 \mathrm{kHz}$ and $3 \mathrm{kHz}$, which are not present in the FIR case. These results suggest the presence of narrowband aliasing errors which, following reconstruction of the signal in the synthesis bank, would give rise to tonal artifacts in the reconstructed residual. 


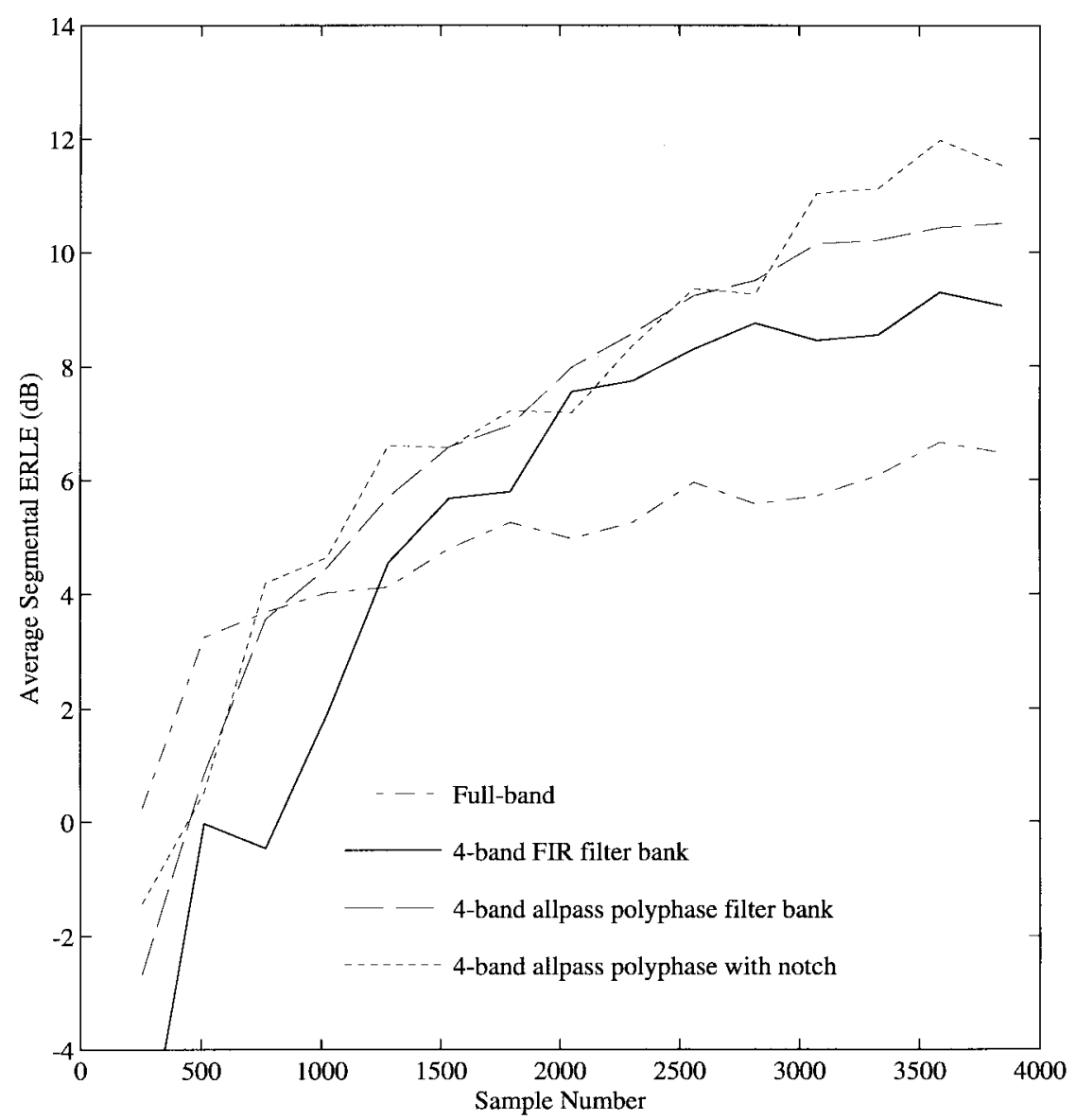

Fig. 13. Averaged segmental ERLE for USASI input signals.

The presence of such artifacts is consistent with the results of Fig. 9 and has been confirmed by listening tests.

It has been shown in Section $\mathrm{V}$ that efficient notch filters can be designed using filter transformations on allpass polyphase filters. Since the aliasing errors in the allpass polyphase IIR case are very narrowband in nature, it is feasible to remove the aliasing without introducing significant spectral distortion through the use of these notch filters at the subband interfaces. An additional motivation for the introduction of notch filters is that they will also attenuate the signal at frequencies where the group delay is largest, thereby reducing the nonlinear phase effects of the IIR filterbank. This notch filtering approach is less attractive in the FIR case, since the aliasing errors are spread broadly over the band and cannot be removed so effectively without introducing relatively large spectral distortions.

To show the effectiveness of the notch filter preprocessing, the previous simulation experiments have been repeated with the addition of notch filters of the type described in Section $\mathrm{V}$ applied to the echo canceller input signals. The results for the four-band case are shown in Figs. 12(d) and 13. The use of the notch preprocessing in the four-band case gives rise to a reduction of around $2 \mathrm{~dB}$ in final misadjustment compared to the IIR case without the notch.

In addition to the above tests, which were conducted using a USASI noise input signal, the echo cancelling schemes have been tested with speech signals recorded from the same hands- free telephone. The segmental ERLE was computed for the four-band case as shown in Fig. 16. This figure also shows in the uppermost plot the amplitude of the signal at the microphone. The utterance text was the French words, "Paris, Bordeaux, Le Mans, Saint-Leu, Leon, Loudun" spoken by a male speaker. Listening tests have also been conducted using these results and it has been verified that the tonal artifacts due to the narrowband aliasing errors in the IIR case are effectively removed by the addition of the notch preprocessing and that no audible distortion due to the presence of the notches in the amplitude spectrum can be detected.

Tests were also performed on an eight-band version of the echo canceller obtained from the four-band version with an additional stage of subband division. By modifying the transformation of (13), replacing $z^{-4}$ with $z^{-8}$, a suitable notch filter can be obtained for use with an eight-band system. The results for the eight-band case are consistent with the four-band results. The lower level of delay introduced by the allpass polyphase IIR approach, compared to the FIR case, is more marked in the eight-band case.

\section{Discussion AND CONCLUSIONS}

This paper has addressed the issue of subband adaptive filtering for applications of acoustic echo control in which the constraints of computational complexity and delay may be difficult to meet with some of the existing subband adaptive 

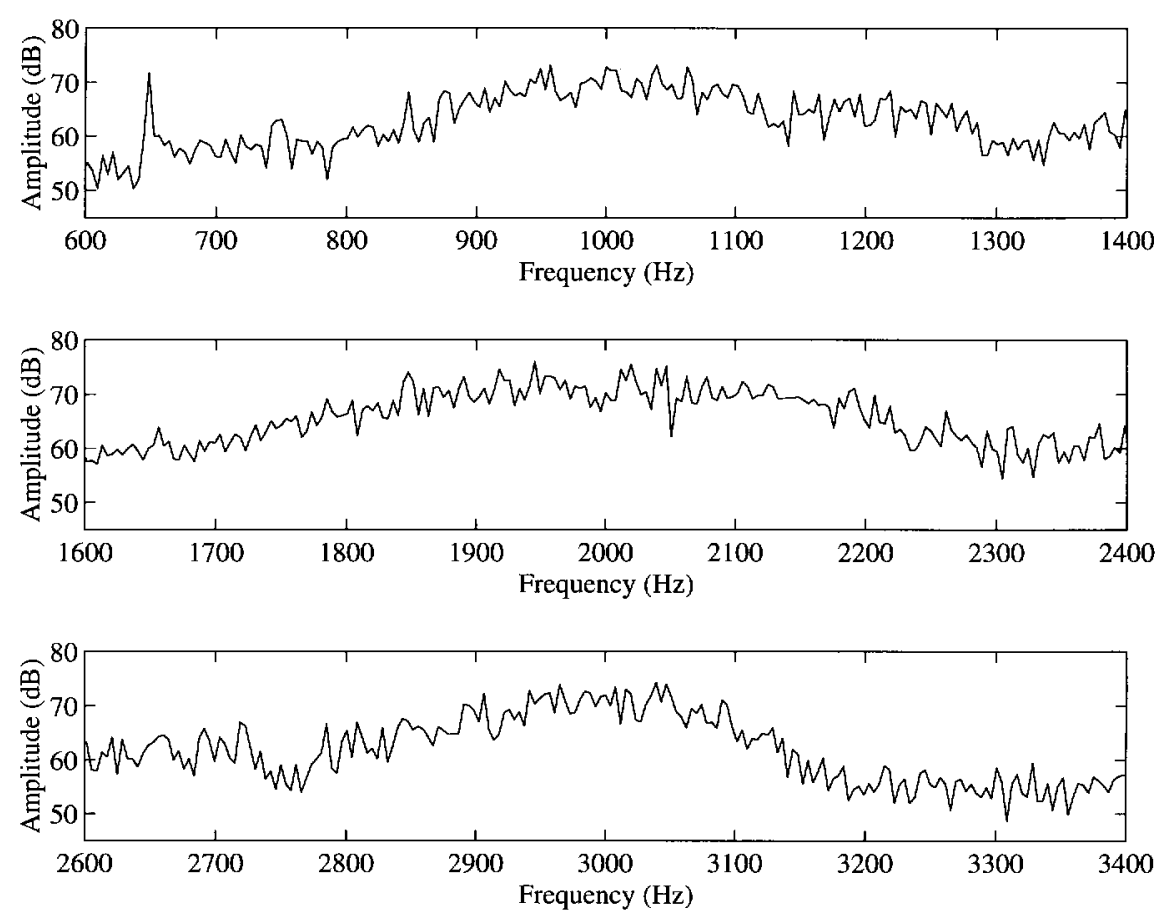

Fig. 14. Subband error signal spectra for the FIR QMF four-band canceller at frequencies near the three band interfaces.
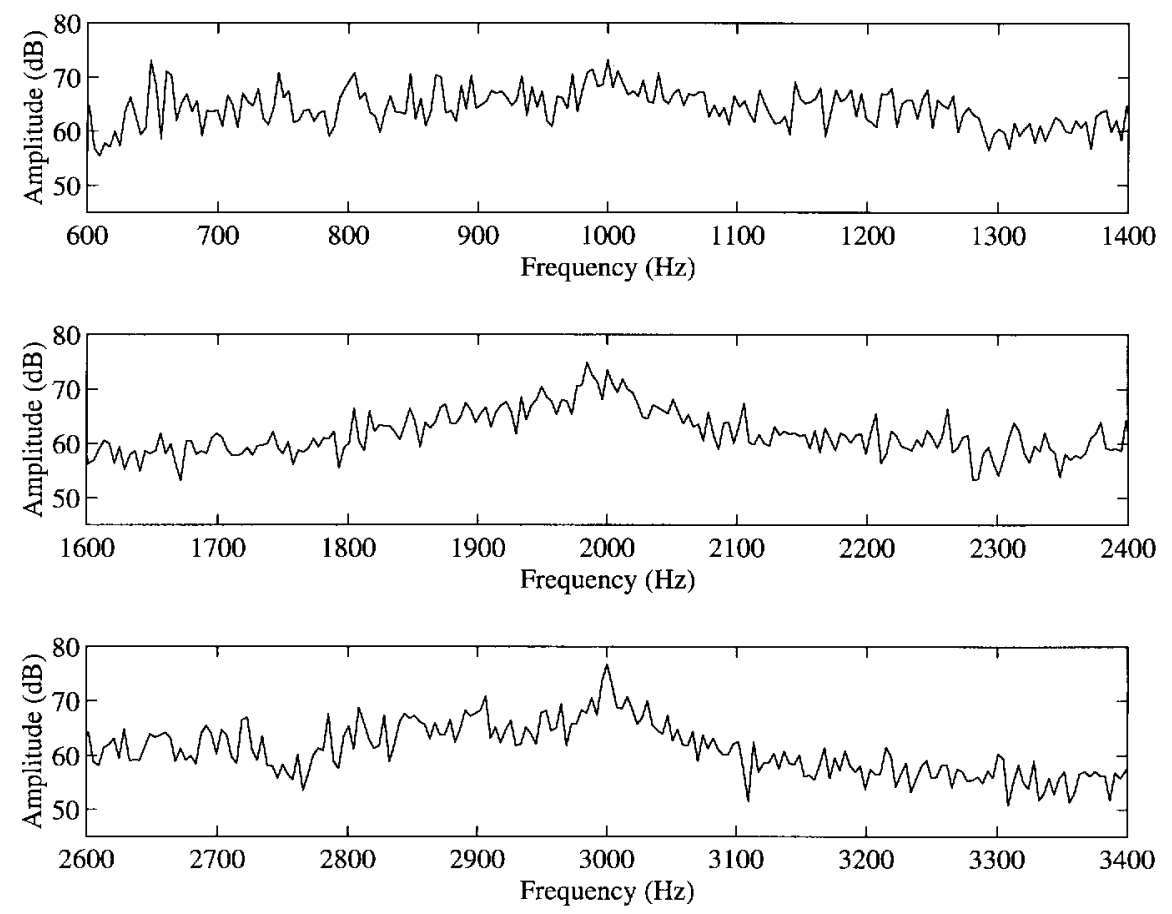

Fig. 15. Subband error signal spectra for the allpass polyphase IIR four-band canceller at frequencies near the three band interfaces.

filtering approaches. It has been observed that the performance of adaptive filtering in the subbands is degraded if the interband discrimination of the filterbank employed is sufficiently poor as to produce significant aliasing components under critical decimation. Filterbanks based on allpass polyphase IIR structures have been introduced as an alternative to the more standard approach involving FIR filterbanks. It has been shown that such multirate systems with very high interband discrimination, low computational cost, and low delay can be built using the allpass polyphase structures and that such filterbanks generate lower level but more coherent aliasing than filters that are less steep. It has been shown that adaptive filters in allpass polyphase filterbank systems perform as well as, but not significantly better than, comparable systems based on FIR filters. A possible explanation for this result is that, although the adaptive filter misadjustment is relatively small at frequencies away from the subband edges, significant misadjustment can be observed at frequencies very close to the subband edges due to the very narrowband nature of the aliasing generated by the sharp allpass polyphase filterbank. 

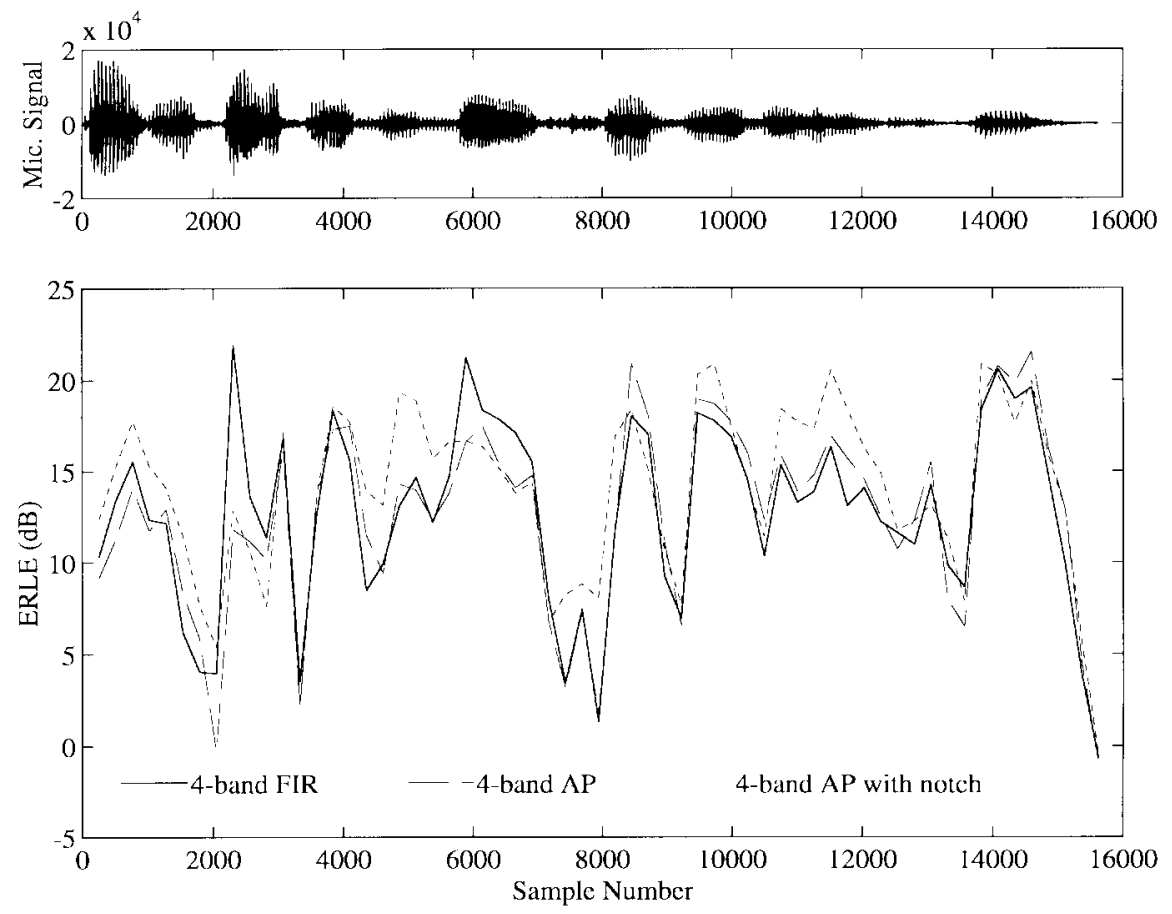

Fig. 16. Echo cancellation results for speech input signal.

Consequently, relatively high peaks in the error signal are obtained at the subband edge frequencies which substantially influence calculations of MSE and give rise to a tonal structure in the residual echo which could be subjectively annoying to users. By imposing spectral nulls in the input signals to the echo canceller at the subband edge frequencies using sharp notch filters, it has been shown that this detrimental effect can be overcome. It has been demonstrated that allpass polyphase IIR filters offer not only a low-cost, low-delay, solution for filterbank implementation but also a good solution for the design of notch prefilters, yielding insignificant spectral distortion in speech. This notch prefiltering would not be appropriate in the FIR case because the aliasing effects are not sufficiently localized in frequency to be removed by notch filters without introducing audible spectral distortions. The simulations performed both with noise signals and with speech have been presented and demonstrate the potential usefulness of the allpass polyphase IIR approach in practical implementations.

\section{ACKNOWLEDGMENT}

The authors wish to thank all the partners of CEC ESPRIT Project 6166 (FREETEL) for their helpful collaboration, and in particular the Speech Processing Group, MATRA Communication, France, for providing the test data used in this paper.

\section{REFERENCES}

[1] A. Gilloire and M. Vetterli, "Adaptive filtering in subbands," in Proc. ICASSP'88, pp. 1572-1575.

[2] _ "Adaptive filtering in subbands with critical sampling: Analysis, experiments, and applications to acoustic echo cancellation," IEEE Trans. Signal Processing, vol. 40, pp. 1862-1875, Aug. 1992.

[3] S. S. Haykin, Adaptive Filter Theory. Englewood Cliffs, NJ: PrenticeHall, 1991.
[4] B. Widrow et al., "Stationary and nonstationary learning characteristics of the LMS adaptive filter," in Proc. IEEE., vol. 64, pp. 1151-1162, Aug. 1976.

[5] P. A. Naylor, J. Alcazar, J. Boudy, and Y. Grenier, "Enhancement of handsfree communications," Ann. Telecommun., vol. 49, pp. 373-379, July-Aug. 1994.

[6] M. Vetterli, "A theory of multirate filterbanks," IEEE Trans. Acoust., Speech, Signal Processing, vol. ASSP-35, pp 356-372, Mar. 1987.

[7] P. P. Vaidyanathan, Multirate Systems and Filter Banks. Englewood Cliffs, NJ: Prentice-Hall, 1993.

[8] A. Gilloire, "Experiments with sub-band acoustic echo cancellers for teleconferencing," in Proc. ICASSP'87, pp. 2141-2144.

[9] W. Kellermann, "Analysis and design of multirate systems for cancellation of acoustic echoes," in Proc. ICASSP'88, pp. 2570-2573.

[10] D. T. M. Slock, "Fractionally-spaced subband and multiresolution adaptive filters," in Proc. ICASSP'91, pp. 2633-2636.

[11] H. Yasukawa, S. Shimada, and I. Furukawa, "Acoustic echo canceller with high speech quality," in Proc. ICASSP'87, pp. 2125-2128.

[12] R. A. Valenzuela and A. G. Constantinides, "Digital signal processing schemes for efficient interpolation and decimation," in Proc. Inst. Elect. Eng., vol. 130, part G, Dec. 1983.

[13] O. Tanrikulu, B. Baykal, J. Chambers, A. G. Constantinides, and P. A. Naylor, "Finite-precision design and implementation of allpass polyphase networks for echo cancellation in subbands," in Proc. ICASSP'95, pp. 3039-3042.

[14] P. A. Naylor and J. E. Hart, "Non-critically decimated subband acoustic echo control using non-critical frequency sampling," in Proc. EUSIPCO'96.

[15] M. Bellanger, G. Bonnerot, and M. Coudreuse, "Digital filtering by polyphase network: Application to sample rate alteration and filter banks," IEEE Trans. Acoust., Speech, Signal Processing, vol. ASSP-24, pp. 109-114, Apr. 1976

[16] R. E. Crochiere and L. R. Rabiner, Multirate Digital Signal Processing Englewood Cliffs, NJ: Prentice-Hall, 1983.

[17] S. K. Mitra and K. Hirano, "Digital all-pass networks," IEEE Trans. Circuits Syst., vol. CAS-21, pp. 688-700, Sept. 1974.

[18] F. Harris, M. d'Oreye de Lantremange, and A. G. Constantinides, "Design and implementation of efficient resampling filters using polyphase recursive allpass filters," in Proc. 25th Asilomar Conf. Signals, Systems, and Computers, 1991, vol. 2, pp. 1031-1036.

[19] J. M. Mendel, "Tutorial on higher-order statistics (spectra) in signal processing and system theory: Theoretical results and some applications," in Proc. IEEE, vol. 79, pp. 278-305, Mar. 1991.

[20] A. Papoulis, Probability, Random Variables, and Stochastic Processes. New York: McGraw-Hill, 1991.

[21] American National Standards Institute, ANSI Std. S1.4-1961. 


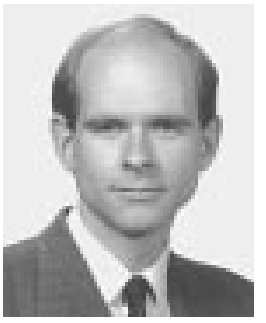

Patrick A. Naylor (M'89) received the B.Eng. (honors) degree in electronic and electrical engineering from the University of Sheffield, U.K., in 1986, and the Ph.D. degree from Imperial College, London, U.K., in 1990.

Since 1989, he has been a Member of the Academic Staff of the Signal Processing and Digital Systems Section, Department of Electrical and Electronic Engineering, Imperial College, where he is also Director of the Information Systems Engineering program. His research interests are in the area of speech signal processing and its applications. He has worked on adaptive signal processing, multirate filterbanks, speaker verification and speech production modeling. He is currently involved in research into both single and multichannel algorithms for acoustic echo and noise control with applications to hands-free telephony and multimedia conferencing systems.

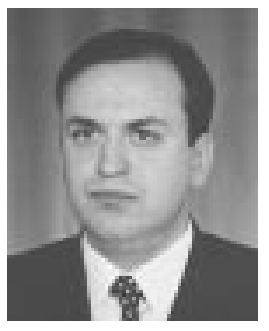

Oğuz Tanrıkulu (S'93-M'95) was born in Ankara, Turkey, in 1969. He received the B.Sc. degree (high honors) in electrical and electronics engineering from the Middle East Technical University (METU), Ankara, Turkey, in 1990, and the M.Sc. and Ph.D. degrees in electrical and electronics engineering from the Imperial College of Science, Technology, and Medicine, London, U.K., in 1991 and 1995 , respectively.

In 1990, he was awarded the British Council scholarship, funded by the Foreign and Commonwealth Office, to pursue the M.Sc. program at Imperial College. In 1992, he was appointed Research Assistant at Imperial College, and until 1995 was involved in the EC ESPRIT Project Enhancement of Hands-Free Telecommunications: FREETEL. Currently, he is a Research Associate at Imperial College working on the EPSRC Multirate Echo Cancellation Research Project. His research interests are in stability and convergence of adaptive algorithms, filterbanks, echo cancellation, and blind equalization for hands-free and wireless systems and optimization theory.

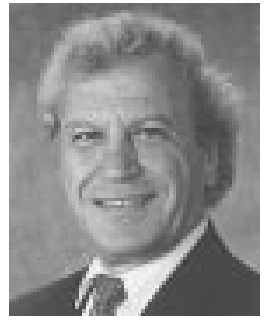

Anthony G. Constantinides (S'68-M'74-SM'78F'98) received the B.Sc. degree in engineering (first class honors) in 1965, and the Ph.D. degree in 1968, both from London University, London, U.K.

$\mathrm{He}$ is Professor of signal processing and the Head of the Signal Processing and Digital Systems Section, Department of Electrical and Electronic Engineering, Imperial College of Science, Technology, and Medicine, London. He has been actively involved with research in various aspects of digital filter design, digital signal processing, and communications for a number of years. His research spans a wide range of digital signal processing, both from the theoretical as well as the practical points of view. His recent work has been directed toward the demanding signal processing problems arising from the area of telecommunications. He has published a range of books and papers in learned journals in the area of digital signal processing and its applications.

Dr. Constantinides served as the first President of the European Association for Signal Processing (EURASIP) and has contributed in this capacity to the establishment of the European Journal for Signal Processing. He has been on, and is currently serving as, a member of many technical program committees of IEEE international conferences. He organized the first ever international series of meetings on digital signal processing, in London initially in 1967, and in Florence, Italy (with Vito Cappellini) since 1972. In 1985, he was awarded the Honor of Chevalier, Palmes Academiques, by the French government, and in 1996, the promotion to Officer, Palmes Academiques. He holds several visiting professorships and other fellowships and honors around the world. $\mathrm{He}$ was recently elected to the Board of Governors of the IEEE Signal Processing Society. He is also serving as a member of the IEEE Signal Processing Society Technical Committee on Neural Networks for Signal Processing. 